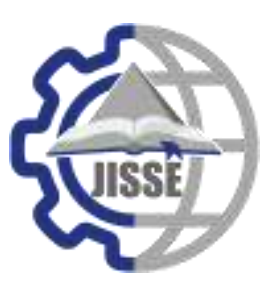

\begin{tabular}{c}
\hline \hline JISSE \\
ISSN: 2636-4425 \\
\hline
\end{tabular}

\title{
Observation of Dual-Peak Signal-to-Noise Ratios from Plasmas Produced in Typical Laser-Induced Breakdown Spectroscopy (LIBS) Experiments
}

\author{
Mohamed A. Khater* and Kamal A. Eid \\ Spectroscopy Department, Physics Division, National Research Centre, El-Buhouth St., Dokki, Giza, Egypt
}

\begin{abstract}
A R T I C LE IN F O
\section{Article history:}

Received: 09-10-2019

Accepted: 01-12-2019

Online: 19-12-2019
\end{abstract}

Keywords:

laser-induced breakdown

spectroscopy, LIBS, signal-to-

noise-ratio, SNR, laser fluence

\begin{abstract}
A B S T R A C T
In this work, emission properties of representative trace and major species in steel plasma plumes produced in typical laser-induced breakdown spectroscopy (LIBS) experiments under standard atmospheric conditions are investigated. The correlation between laser fluence and spatially integrated measurements of emission characteristics of the plasma at a typical delay time of $2 \mu \mathrm{s}$ from the laser pulse onset is thoroughly explored. Q-switched neodymium-doped yttrium aluminum garnet (Nd: YAG) laser emitting at its fundamental wavelength of $1064 \mathrm{~nm}$ was employed to generate the plasmas under investigation. The results showed that the signal-to-noise ratio of all lines investigated exhibit irregular behaviour in which two maxima can be identified at specific laser fluence values. This behaviour is attributed to lower standard deviation of the background intensity indicating stable plasma conditions relevant for spectrochemical analysis. Plasma shielding of the incident laser energy, due to electron-atom inverse bremsstrahlung (IB), becomes more critical beyond laser fluencies larger than about $45 \mathrm{~J} \mathrm{~cm}^{-2}$.
\end{abstract}

\section{Introduction}

Spectral emission data recorded from laser-produced plasma sources are a precious mine of information. For example, line intensities have long been exploited for the rapid elemental identification and quantification of all materials. Moreover, intensities, profiles as well as spectral shifts of carefully selected lines are widely used for determination of the main emitting plasma properties namely electron temperature and density. The instrumental technique normally employed to carry out such studies is generally called laser-induced breakdown spectroscopy (LIBS) [1], [2], but also occasionally termed laser-induced plasma spectroscopy (LIPS) [3]. LIBS has recently reached a maturity state due to significant technological advances in its main components including laser and detection system, enabling a wide range of applications in a variety of fields. Examples include biomedical/biological specimens [4], [5], [6], cultural heritage restoration [7], [8], environmental analysis [9], [10], [11], [12], explosive materials [13], metals and alloys [14], [15], [16], [17], [18], nuclear fusion [19], polymeric materials [20], [21],

*Mohamed A. Khater, Spectroscopy Department, National Research Center, Giza, Egypt, +201273792774, drmakhater@gmail.com space exploration [22], and underwater objects, [23].

The determination sensitivity of species emitted in the plasma is dependent on the correlation between their spectral signal and background intensities represented in signal-to-background (SBR) and signal-to-noise ratios (SNR) parameters. In turn, these parameters strongly rely on plasma-producing laser source conditions including pulse energy, wavelength, and pulse duration, as well as ambient atmosphere compositions and pressures. A usual routine therefore has been the careful optimization of some, or all variables related to a specific LIBS application to achieve the best possible results in terms of the experiments' figures of merit. The SBR, and hence precision and detection limit, of a singly-ionized carbon spectral line in plasmafacing wall materials was improved upon optimizing a number of key parameters including laser pulse energy (250 to $600 \mathrm{~mJ}$ ) using a newly-designed LIBS system [24]. Optimum laser energy value of $450 \mathrm{~mJ}$ was selected for this work. A comparison between IR and UV laser wavelengths and energies (up to $100 \mathrm{~mJ}$ ) for the correlation between plasma characteristics and physical properties of six metal targets $(\mathrm{Pd}, \mathrm{Mo}, \mathrm{Ni}, \mathrm{Cu}, \mathrm{Co}$, and $\mathrm{Au}$ ) was conducted in air at atmospheric pressure using time-resolved 
emission spectroscopy (up to $32 \mu \mathrm{s}$ delay) [25]. The SBR increased with time delay then maximized at longer delays in plasmas produced by IR laser compared to UV laser at the same laser pulse energy. Cabalín and Laserna [26] reported an extensive study on the nanosecond laser induced breakdown threshold of nine different metals $\mathrm{Zn}, \mathrm{Al}, \mathrm{Ag}, \mathrm{Cu}, \mathrm{Ni}, \mathrm{Fe}, \mathrm{Cr}, \mathrm{Mo}$, and $\mathrm{W}$ ) by recording SNRs of their corresponding neutral spectral lines in plasmas generated by ultraviolet, visible as well as infrared laser wavelengths at atmospheric air pressure. The authors demonstrated that short laser wavelengths facilitate better ablation of metals, while IR wavelengths permit more efficient inverse bremsstrahlung (IB) process (laser-plasma coupling) making them most suitable for various LIBS metal applications in atmospheric air environment.

The enhancement of LIBS sensitivity for spectrochemical analysis of steel has been recently reviewed [27]. Zhang and coauthors [28] determined accuracy, precision, and detection limits of seven elements in steel reference materials by optimizing laser energy, lens-to-sample distance, and delay time. Most recently, a comparative study between picoseconds (ps) and nanosecond (ns) laser pulses for LIBS measurements on steel samples was recently conducted [29]. Better SBRs for several atomic and ionic lines, as well as improved precision and limits of detection for the elements $\mathrm{Fe}, \mathrm{Cr}$, and $\mathrm{Si}$ were obtained with the ps laser.

In the present work, for the first time, irregular SNR behavior with respect to laser energy fluence typically used in LIBS applications is reported. Several spectral lines representing trace as well as major metals emitted in laser-produced steel plasma generated in standard atmospheric conditions were investigated. All spectra were spatially integrated but recorded at $2 \mu$ s delay from the onset of the incoming laser pulse as a function in the fluence range $10-69 \mathrm{~J} \mathrm{~cm}^{-2}$.

\section{Experimental}

A schematic diagram for the LIBS system employed in the present work is depicted in Figure 1.

\subsection{The Laser Beam Focusing Conditions}

The laser is a Q-switched Nd:YAG (Innolas Laser, model Spit Light Compact 400) type with maximum output pulse energy of $(400 \pm 3) \mathrm{mJ}, 6 \mathrm{~ns}$ pulse width with jitter less than $1 \mathrm{~ns}, 10 \mathrm{~Hz}$ repetition rate, and emission wavelength of $1064 \mathrm{~nm}$. The 6-mm in diameter laser beam is directed downward using a $45^{\circ}$ mirror with relevant optimum reflectivity. A Galilean beam expander was then used to stretch the laser beam to a diameter of $20 \mathrm{~mm}$, and a plano-convex lens with $80 \mathrm{~mm}$ focal length is employed to focus the laser beam onto a low alloy steel target in air at atmospheric pressure within a custom-made target chamber (not shown). The calculated diffraction limited radius of the focused laser beam, corrected to a Gaussian laser beam profile, was about $6 \mu \mathrm{m}$.

In the present work, however, the low-alloy steel target was always kept at a distance of about $1.5 \mathrm{~mm}$ ahead the nominal focus of the laser beam (i.e., the focal plane is located inside the target surface) in order to prevent accidental air breakdown induced in front of the target surface, which essentially contributes to decreasing the laser-target coupling and hence ablation efficiency. Accordingly, the radius of the laser beam on the target was estimated to be about $400 \mu \mathrm{m}$. At the nominal maximum laser energy of $400 \mathrm{~mJ}$, the target then receives a maximum laser pulse irradiance of about $13 \mathrm{GW} / \mathrm{cm}^{2}\left(80 \mathrm{~J} / \mathrm{cm}^{2}\right)$. Gibson et al. [30] stated that at laser intensities larger than several order of $10^{8} \mathrm{~W} / \mathrm{cm}^{2}$, ejection of macroscopic particles from a solid target is minimal, which implies that the composition of the plasma plume is representative of the sample material. This signifies an ideal situation for spectrochemical analysis.

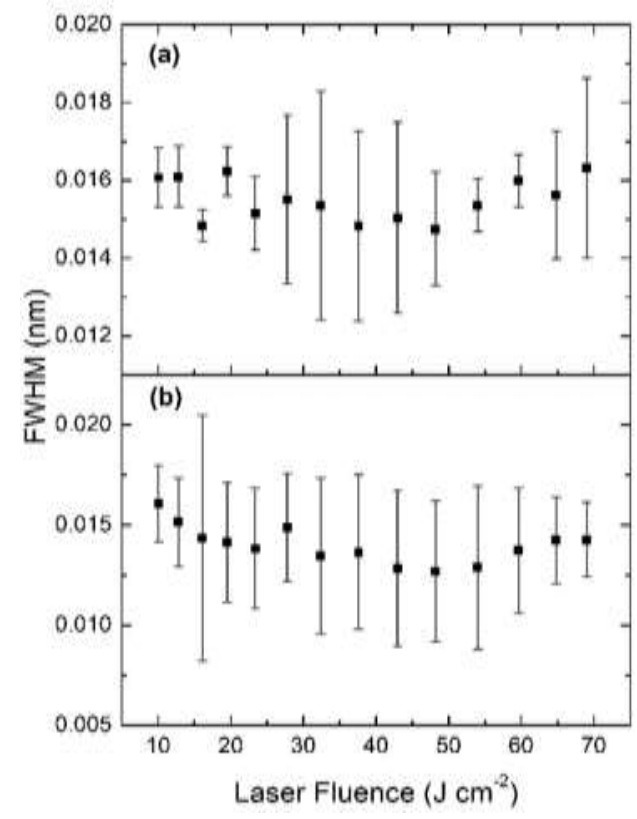

Figure 1: Schematic Diagram for Experimental Set-up

\subsection{Emission Collimation and Detection}

The radiation emitted from the laser-generated steel plasma is collected by a suitable lens and then guided through an attached optical fiber cable, which is in turn connected to 10 $\mu \mathrm{m} \times 30 \mu \mathrm{m}[\mathrm{W} \times \mathrm{H}]$ entrance slit of an EMU-120/65 echelle spectrograph (Catalina Scientific Instruments) with $120 \mathrm{~mm}$ collimator mirror. The spectrograph is also equipped with UVU3 (UV/VIS/NIR) cassette completed with an aperture stop of 20 $\mathrm{mm}$ in diameter.

The dispersed light is detected by an intensified front illuminated Electron Multiplying CCD EMCCD (Andor Technology, model iXon 3 885) with $1004 \times 1002$-pixel array, and $8 \mu \mathrm{m} \times 8 \mu \mathrm{m}$ pixel size. The CCD array has a nominal quantum efficiency of $65 \%$ at $600 \mathrm{~nm}$. The nominal resolution of the detection system (spectrograph+ EMCCD) is about $0.02 \mathrm{~nm}$. For all measurements, the exposure time (gate width) of the EMCCD detector was set at an optimum value of $80 \mu \mathrm{s}$.

All experiments were performed using standard disc-shaped (38 $\mathrm{mm}$ in diameter, and $19 \mathrm{~mm}$ in height) low-alloy steel sample SS-CRM 402/2 (Bureau of Analyzed Samples LTD, UK), with nominal iron mass content of about $97 \%$. The steel target is mounted on a 3 -axis computer controlled translational stages 
with $75 \mathrm{~mm}$ linear travel and $12.5 \mu \mathrm{m}$ linear resolution. Every measurement was recorded by firing 30 successive laser shots at $10 \mathrm{~Hz}$ repetition rate onto a specific position of the target surface, preceded by 10 pre-shots for adequate surface cleaning. In addition, measurements were repeated 3 times at different locations on the target surface. The propagation of error method was used to calculate the standard deviation of all measuring quantities.

\section{Results}

\subsection{Spectral Lines Selection}

Spectroscopic information of lines belonging to the elements under investigation was taken from NIST's atomic spectra database and is presented in Table 1. All selected lines were ensured to be spectral-interference free. Moreover, spectral lines for iron are non-resonant lines, while those for the trace elements Aluminum and Copper are the lines normally used for determination of their content in iron-based materials. A further check on the suitability of selected lines, including those representing iron was performed by recording the full width at half maximum (FWHM) of their Lorentzian profiles as a function of the laser fluence in order to ensure that they are self-absorption free. An example of the results obtained for the lines Al I 396.15 $\mathrm{nm}$ and Fe I $397.77 \mathrm{~nm}$ are depicted in Figure 2, where it is clear that the FWHM is not an increasing function of the laser fluence for the two lines, within the uncertainty of the experimental measurements.

Table 1: Spectroscopic data for the elements under investigation

\begin{tabular}{|c|c|c|c|c|}
\hline $\begin{array}{l}\text { Emitting } \\
\text { Species }\end{array}$ & $\begin{array}{l}\text { Wavelength } \\
(\mathbf{n m})\end{array}$ & $\begin{array}{l}\text { Lower } \\
\text { Energy } \\
\text { Level } \\
(\mathrm{eV})\end{array}$ & $\begin{array}{l}\text { Higher } \\
\text { Energy } \\
\text { Level } \\
(\mathrm{eV})\end{array}$ & $\begin{array}{l}\text { Transition } \\
\text { Probability } \\
\left(\mathbf{s}^{-1}\right)\end{array}$ \\
\hline Fe I & 397.77 & 1.53 & 3.69 & $6.4 \times 10^{6}$ \\
\hline Al I & 396.15 & 0.01 & 3.14 & $9.8 \times 10^{7}$ \\
\hline Cu I & 327.4 & 0.00 & 3.79 & $1.4 \times 10^{8}$ \\
\hline
\end{tabular}

\subsection{Effect of Laser Fluence}

In the present work, the effective laser fluence was varied from about $10 \mathrm{~J} \mathrm{~cm}^{-2}$ to $69 \mathrm{~J} \mathrm{~cm}^{-2}$ (corresponding to laser energy from $50 \mathrm{~mJ}$ to $345 \mathrm{~mJ}$ ) by simply changing the delay time between flash lamps and the pockels cells onsets at fixed delay time for integration and gate width of $2 \mu \mathrm{s}$ and $80 \mu \mathrm{s}$, respectively. Each laser energy value was measured at the sample position, not at the laser output aperture, using a calibrated joule meter.

Figure 3 is an example of a portion of the LIBS spectrum recorded at different laser fluences, demonstrating the spectral intensity evolution of the Al I $396.15 \mathrm{~nm}$ and Fe I $397.77 \mathrm{~nm}$ lines. General outlook at the figure reveals that the spectral line intensity is an obvious increasing function of laser beam fluence, as one might expect. The intensity of a spectral line, integrated over the line profile $\mathrm{L}$, corresponding to the transition $\mathrm{q} \rightarrow \mathrm{p}$ is given by equation (1), where $\varepsilon$ is the emission coefficient of the spectral line undergoing the transition, $\mathrm{A}$ is the corresponding

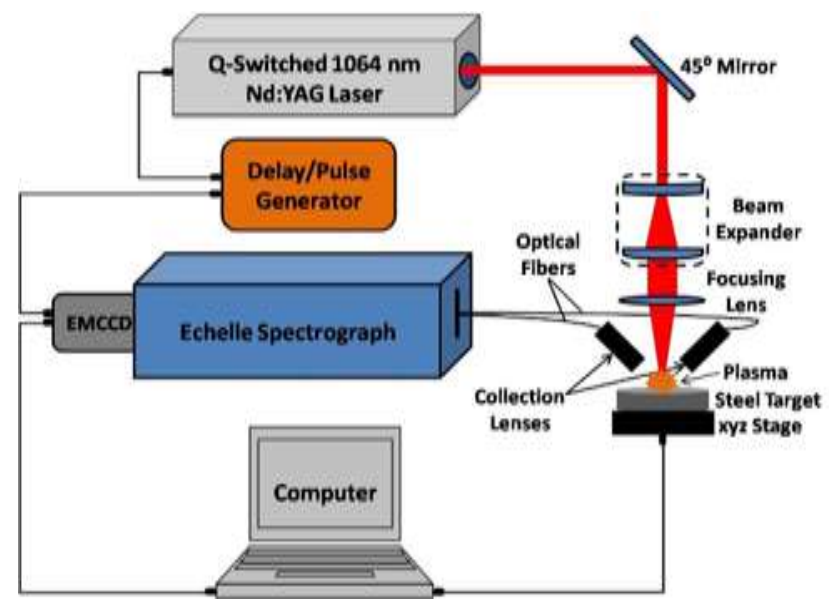

Figure 2: FWHM versus Laser Fluence. (a) Al I 396.15 nm, (b) Fe I 397.77 nm

Transition probability and $\mathrm{h}$ is Planck's constant. If the energy levels concerned are in local thermodynamic equilibrium (LTE).

$$
I=\int_{\text {Line }} \varepsilon_{v, L} d v=\frac{h v_{p q}}{4 \pi} A_{q p} N_{q}
$$

Then the population density of the upper level, $\mathrm{N}_{\mathrm{q}}$, is related to the ground level number density, $\mathrm{N}_{\mathrm{T}}$, by the Boltzmann equation:

$$
\frac{\mathrm{N}_{\mathrm{q}}}{\mathrm{N}_{\mathrm{T}}}=\frac{\mathrm{g}_{\mathrm{q}}}{\mathrm{Q}\left(\mathrm{T}_{\mathrm{exc}}\right)} \exp \left(-\frac{\mathrm{E}_{\mathrm{q}}}{\mathrm{k}_{\mathrm{B}} \mathrm{T}_{\mathrm{exc}}}\right)
$$

where $g_{q}$ and $E_{q}$ are the statistical weight and energy of the upperlevel q, respectively; $Q$ is the partition function of the relevant species; $\mathrm{k}_{\mathrm{B}}$ is Boltzmann's constant and $\mathrm{T}_{\mathrm{exc}}$ is the plasma excitation temperature.

From the above two equations, we get an expression for the spectral line intensity in which a proportionality with the plasma excitation temperature is obvious:

$$
I=\frac{h v_{p q}}{4 \pi} A_{q p} \frac{N_{T} g_{q}}{Q\left(T_{e x c}\right)} \exp \left(-\frac{E_{q}}{k_{B} T_{e x c}}\right)
$$

Colombant and Tonon [31] related $\mathrm{T}_{\mathrm{exc}}(\mathrm{eV})$ to the incident laser power density, $\phi\left(\mathrm{W} \mathrm{cm}^{-2}\right)$ such that:

$$
\mathrm{T}_{\mathrm{e}} \approx 5.2 \times 10^{-6} \mathrm{Z}^{1 / 5}\left[\lambda^{2} \varphi\right]^{3 / 5}
$$

Where, $\mathrm{Z}$ is the atomic number of the element under investigation. Accordingly, the spectral line intensity is also a function of the 
laser power density, and hence the laser fluence.

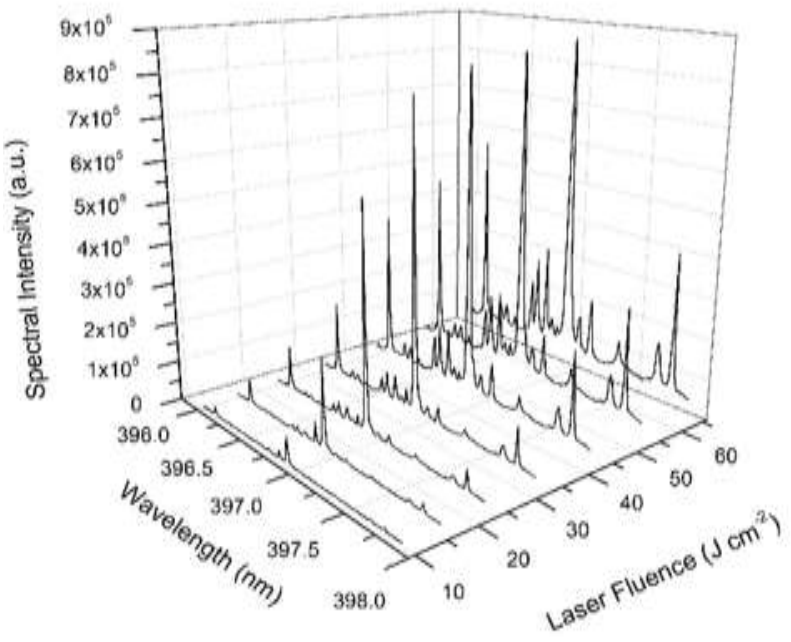

Figure 3: 3D Plot of Laser-Induced Steel Plasma Spectrum in the Range 396$398 \mathrm{~nm}$

Figure 4 confirms this relationship by showing that the intensity of the lines increases monotonically with laser fluence until a plateau is reached at relatively high laser fluence, where the plasma starts to effectively blocks larger portion of the laser beam from reaching the target surface and ablate extra material.

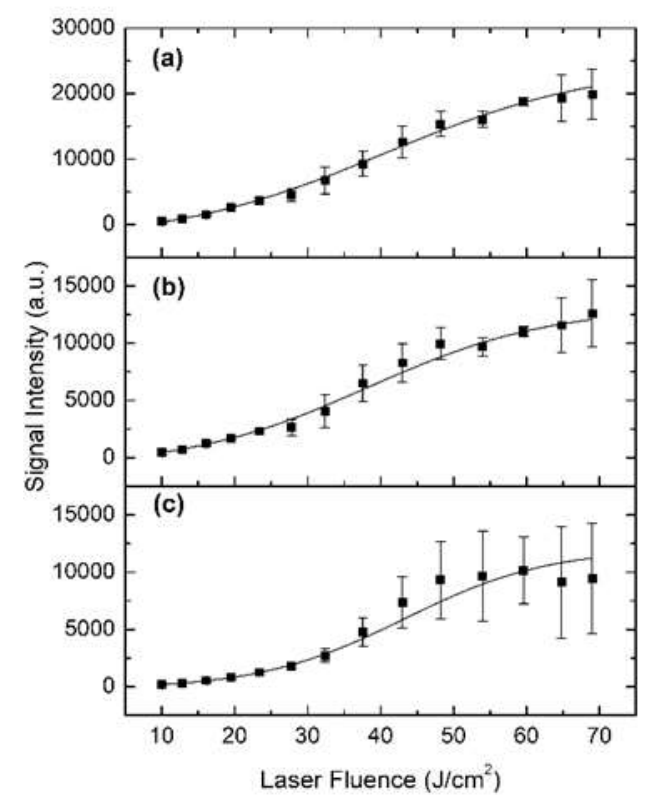

Figure 4: Dependence of Net Signal Intensity on Laser Energy Fluence for (a) Cu I 327.4 nm, (b) Al I 396.15 nm, and (c) Fe I 397.77 nm. Solid Squares Represent Experimental Data; Solid Lines Represent Fitting with Boltzmann Function

The laser fluence value at which this effect starts to critically function can be inferred from Figure 5 in which a plot between the SBR and laser fluence was constructed. The SBR firstly increases with laser fluence, reaches a maximum at almost the same value for all 3 lines under investigation, before it levels off up to the maximum points of our experiments at $69 \mathrm{~J} \mathrm{~cm}^{-2}$ within limits of measurements' uncertainty. The optimum value for SBR occurs around $45 \mathrm{~J} \mathrm{~cm}^{2}$, which also marks the value after which the plasma shielding effect becomes more important.

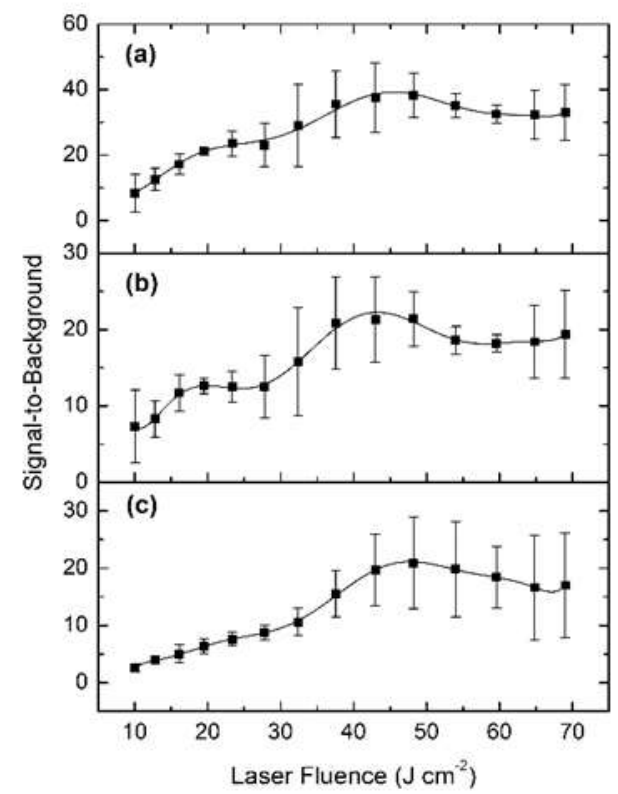

Figure 5: Dependence of SBR on Laser Energy Fluence for (a) Cu I 327.4 nm, (b) Al I $396.15 \mathrm{~nm}$, and (c) Fe I $397.77 \mathrm{~nm}$. Solid Squares Represent Experimental Data; Solid Lines Represent Fitting with Polynomial Function

Figure 6 demonstrates an example of Boltzmann plot used for evaluation of excitation temperature of the plasma source under consideration. In the graph, the intensity function of selected 14 iron spectral lines is plotted against their upper level energy. The excitation temperatures deduced from such plots were plotted as a function of laser fluence and the results are depicted in Figure 7. As can be seen, $T_{\text {exc }}$ is a weak function of laser fluence within limitations of the experiments' uncertainty. In fact, $\mathrm{T}_{\mathrm{exc}}$ varied from about $19400 \pm 3600 \mathrm{~K}$ at $10 \mathrm{~J} \mathrm{~cm}^{-2}$, to about $20800 \pm 7100 \mathrm{~K}$ at $69 \mathrm{~J} \mathrm{~cm}^{-2}$. Accordingly, we cannot attribute the increase in spectral line intensity to a corresponding rise in excitation temperature. Rather, the enhancement effect must be due to an increase in materials ablated from the target surface at lower to medium laser fluences, and then becomes more or less constant because of the effect of plasma shielding in which larger portions of the incident laser photons are gradually absorbed due to electron-atom inverse bremsstrahlung (IB).

The increase in IB effect may be confirmed by studying the evolution of the plasma electron number density with laser fluence. Electron density of the plasma is estimated using the iron spectral line at $538.34 \mathrm{~nm}$ [32], which has a large Stark width. The line intensity profile of this line is shown in Figure 8 . The profile was fitted to Voigt function with fixed Gaussian width due to instrumental broadening. In turn, the instrumental width was found to be a pure Gaussian using the Fe I $532.80 \mathrm{~nm}$ recorded at delay time of $100 \mu \mathrm{s}$ where the contribution from Stark broadening is negligible. 


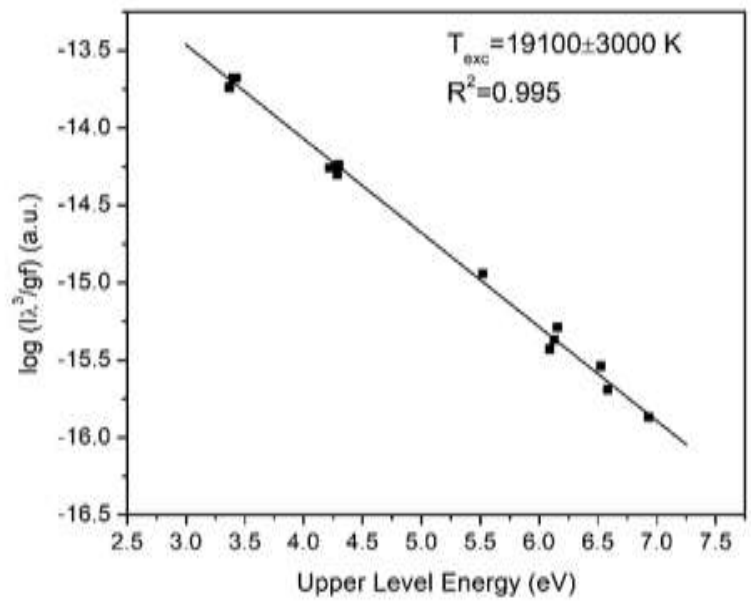

Figure 6: Boltzmann Plot for 14 Fe I Spectral Lines in the Region $365-376$ nm. Laser Fluence is $32.4 \mathrm{Jcm}^{-2}$. Solid Squares Represent Experimental Data; Solid Line Represents Linear Fitting

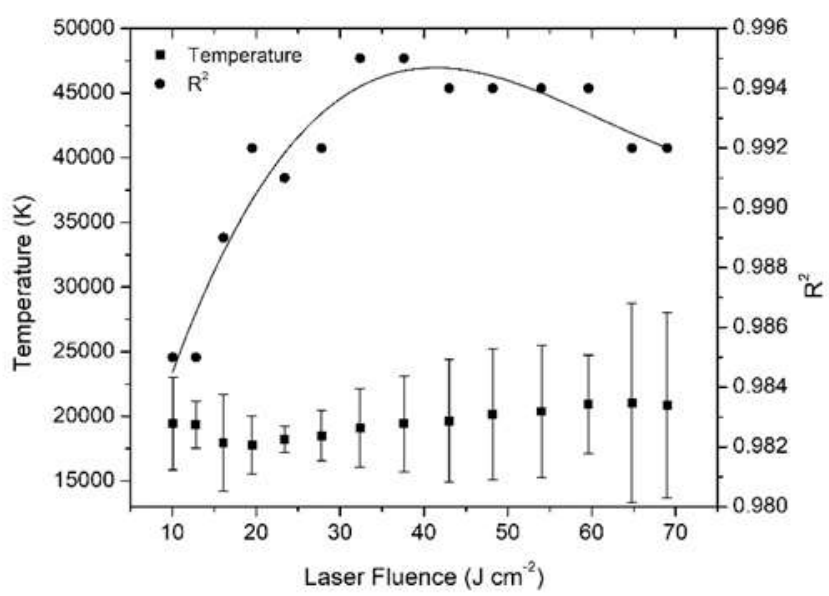

Figure 7: Plasma Excitation Temperature (solid squares) and Correlation Coefficient Fitting to Boltzmann Plots (solid circles) as a Function of Laser Energy Fluence. Solid Line Represents Fitting with Polynomial Function

Figure 9 illustrates the variation of electron density with laser fluence. The electron density is constant up to a fluence of $45 \mathrm{Jcm}^{-}$ before it undergoes an abrupt gradual increase up to the last laser fluence of $69 \mathrm{~J} \mathrm{~cm}^{-2}$. It is also clear that the quality of the fitting function noticeably increases at higher values of laser fluence.

\subsection{The Signal-to-Noise Ratio (SNR)}

The measurement of elemental spectral signals as well as corresponding background continuum intensities as a function of various experimental parameters including laser pulse energy is critical for all LIBS applications. For example, the signal-tobackground ratio (SBR) is a decisive parameter in selecting the most prominent spectral line/s belonging to the same species relevant for a application.

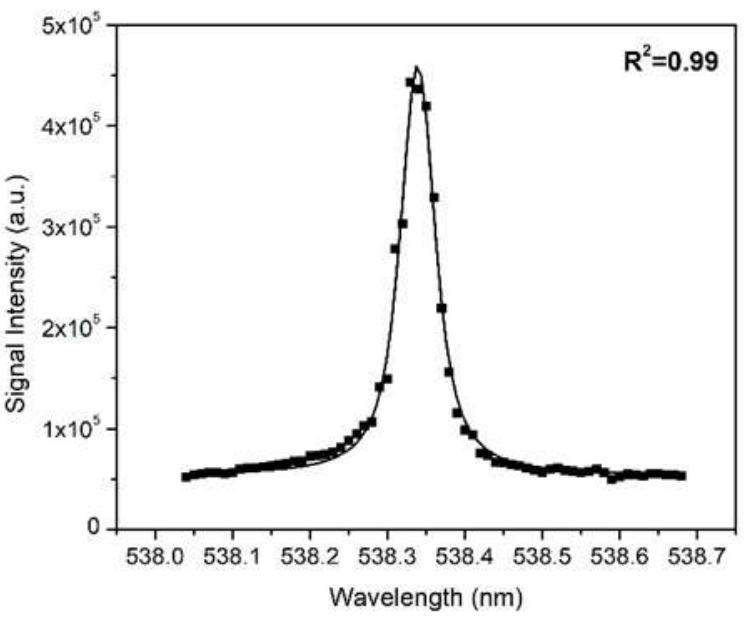

Figure 8: Spectral Profile of the Fe I $538.34 \mathrm{~nm}$ Line. Solid Squares Represent Experimental Data; Solid line Represents Fitting with Voigt Function

In addition, it determines a set of optimum experimental conditions at which measurements should be carried out to get the best results in terms of analytical figures of merit. SBR is defined as the ratio between the net signal intensity and the background intensity:

$$
\operatorname{SBR}=\frac{I_{s}-I_{b}}{I_{b}}
$$

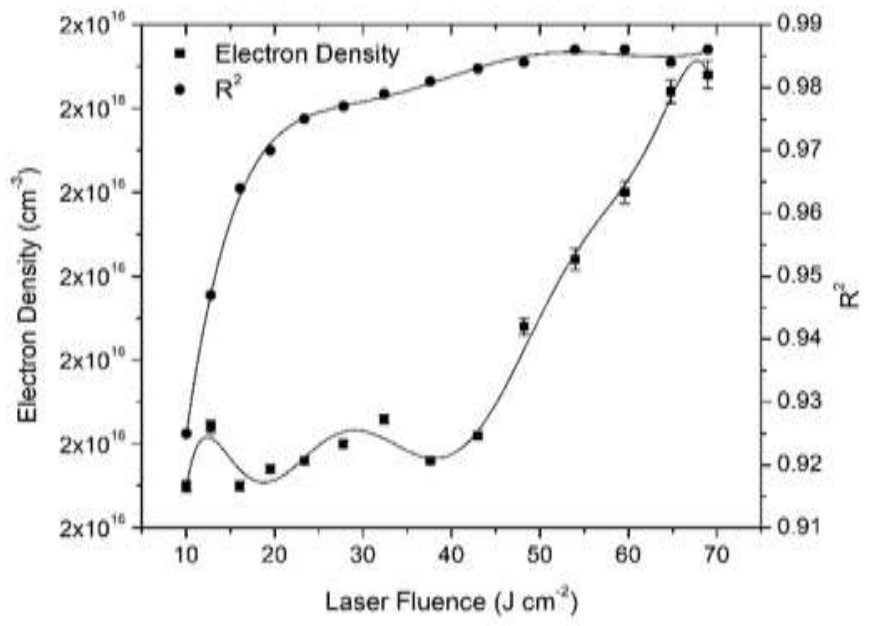

Figure 9: Plasma Electron Density (solid squares) and Correlation Coefficient Fitting to Voigt Function (solid circles) versus Laser Energy Fluence. Solid Lines Represent Fitting with Polynomial Functions

On the other hand, the signal-to-noise ratio (SNR) of a spectral line representing an element is defined as the net signal intensity divided by the fluctuation in the undelaying background continuum:

$$
\operatorname{SNR}=\frac{\mathrm{I}_{\mathrm{s}}-\mathrm{I}_{\mathrm{b}}}{\sigma_{\mathrm{I}_{\mathrm{b}}}}
$$

Once the most prominent SBR-based spectral lines are selected, a comparison relying on their SNR values is performed 
in order to get the best determination sensitivity of the relevant species in the plasma. It is worth noting that the determination sensitivity (limit of detection, LOD) of an element depends strongly on SNR [33]:

$$
L O D=\frac{3.3 \sigma_{I_{b}}}{S}
$$

where: $\mathrm{S}$ is the slope of the analytical calibration curve. It is obvious that the lower fluctuation in background intensity measurements results in concomitant lower or better determination sensitivity of the relevant element.

Figure 10 demonstrates the dependence on laser fluence of the signal-to-noise ratio (SNR) as well as background intensity for the spectral lines of trace elements $\mathrm{Cu}$ and $\mathrm{Al}$. For the fluence range studied, two distinctive peaks can be identified at specific values of laser fluence; the first maximum occurs at around 20 $\mathrm{Jcm}^{-2}$, while the other peak is located further at about $60 \mathrm{Jcm}^{-2}$.

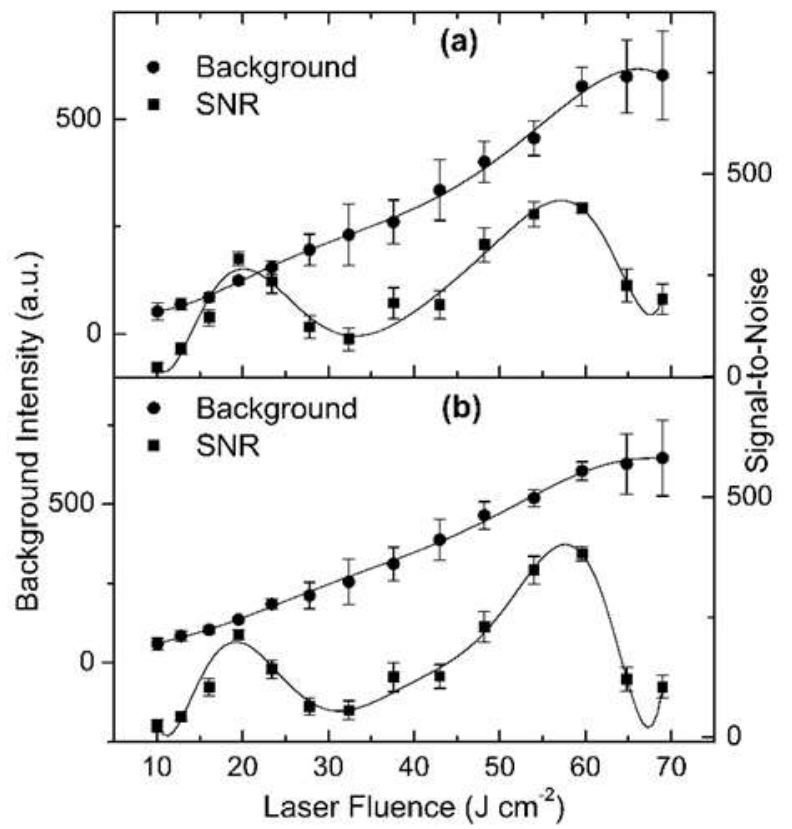

Figure 10: Dependence of SNR and Background Intensity on Laser Energy Fluence for (a) Cu I 327.4 nm, and (b) Al I 396.15 nm. Solid Lines Represent Fitting with Polynomial Function

The standard deviation of the background measurements, as indicated by the extension of the corresponding error bars, is remarkably low at these specific laser fluence values indicating higher SNRs. It is also observed that the standard deviation of intensity as well as SBR shown in figures 4 and 5, respectively, are relatively better around these laser fluences compared to other values. These observations indicate that the plasma at these laser fluence values exhibit a state of stable conditions for spectrochemical analysis, with laser fluence at around $60 \mathrm{~J} \mathrm{~cm}^{-2}$ more favorable than that at $20 \mathrm{~J} \mathrm{~cm}^{-2}$.

\section{Conclusion}

In the present work, spatially-integrated spectral emission parameters including signal and background intensities, as well as SBR and SNR of representative trace and major elements in steel plasmas induced by $1064 \mathrm{~nm}$ laser pulses at standard atmospheric conditions as functions of the effective laser energy density (Laser fluence) were recorded. Both signal as well as background intensities of spectral lines under consideration increase monotonically with laser fluence up to $45 \mathrm{Jcm}^{-2}$, beyond which a noticeable saturation was observed due to formation of absorbing plasma of the incident laser energy. Evaluation of excitation temperature of the plasma indicated that the rise in signal intensity is not due an increase in temperature, but rather because of a corresponding increase in mass ablation rate up to $45 \mathrm{Jcm}^{-2}$. The absorption of the laser beam energy in the plasma beyond this laser fluence was confirmed by estimating its electron density that demonstrated a significant increase beyond this value. SNR of all lines peaked at two specific laser fluence values indicating stable plasma conditions suitable for spectrochemical analysis, with the greater maximum at about 60 $\mathrm{Jcm}^{-2}$ compared to the other peak at around $20 \mathrm{Jcm}^{-2}$. This anomalous behavior was attributed to the exceptionally low fluctuations in the emission parameters of the plasma generated at these values.

\section{Conflict of Interest}

The authors declare no conflict of interest.

\section{References}

[1] D. M. Wong, A. A. Bol'shakov, R.E. Russo, Laser Induced Breakdown Spectroscopy, in Encyclopedia of Spectroscopy and Spectrometry, edited by J. C. Lindon, G. E. Tranter, D. W. Koppenaal, Academic Press, 2017.

[2] S. Musazzi, U. Perini, Laser Induced Breakdown Spectroscopy: Theory and Applications, Springer, 2014.

[3] M. A. Khater, P. van Kampen, J. T. Costello, J.-P. Mosnier, E. T. Kennedy, "Time-integrated laser-induced plasma spectroscopy in the vacuum ultraviolet for the quantitative elemental characterization of steel alloys" $\mathrm{J}$ Phys. D: Appl. Phys.,33(18), 2252-2262, 2000. https://doi.org/10.1088/0022-3727/33/18/308

[4] S. A. Davari, S. Masjedi, Z. Ferdous, D. Mukherjee, "In-vitro analysis of early calcification in aortic valvular interstitial cells using Laser-Induced Breakdown Spectroscopy (LIBS)" J. Biophotonics,11(1), 1-10, 2017. https://doi.org/10.1002/jbio.201600288

[5] S. Zivkovic, M. Momcilovic, A. Staicu, J. Mutic, M. Trtica, J. Savovic, "Spectrochemical analysis of powdered biological samples using transversely excited atmospheric carbon dioxide laser plasma excitation" Spectrochim. Acta Part B,128, 22-29, 2017. https://doi.org/10.1016/j.sab.2016.12.009

[6] Y. Moon, J. H. Han, S. Shin, Y.-C. Kim, S. Jeong, "Elemental analysis of tissue pellets for the differentiation of epidermal lesion and normal skin by laser-induced breakdown spectroscopy" Biomed. Opt. Exp. 7(5), 1626-1636, 2016.

https://doi.org/10.1364/BOE.7.001626

[7] V. Tankova, G. Malcheva, K. Blagoev, L. Leshtakov, "Investigation of archaeological metal artefacts by laser-induced breakdown spectroscopy (LIBS)" in 2018 20th International Summer School on Vacuum, Electron,and Ion Technologies, Sozopol, Bulgaria, 2017. https://doi.org/10.1088/1742-6596/992/1/012003

[8] G. S. Senesi, I. Carrarab, G. Nicolodellic, D. M. B. P. Miloric, O. De Pascale, "Laser cleaning and laser-induced breakdown spectroscopy applied in removing and characterizing black crusts from limestones of Castello Svevo, Bari, Italy: A case study" Microchem. J., 124, 296-305, 2016. https://doi.org/10.1016/j.microc.2015.09.011

[9] J. Singh, R. Kumar, S. Awasthi, V. Singh, A. K. Rai, "Laser Induced breakdown spectroscopy: A rapid tool for the identification and 
quantification of minerals in cucurbit seeds" Food Chemistry, 221, 1778$1783,2016$.

https://doi.org/10.1016/j.foodchem.2016.10.104

[10] S. M. Zaytsev, I. N. Krylov, A. M. Popov, N. B. Zorov, T. A. Labutin, "Accuracy enhancement of a multivariate calibration for lead determination in soils by laser induced breakdown spectroscopy" Spectrochim. Acta Part B, 140, 65-72, (2018).

https://doi.org/10.1016/j.sab.2017.12.005

[11] A. P. M. Michel, F. Sonnichsen, "Laser induced breakdown spectroscopy for heavy metal detection in a sand matrix" Spectrochim. Acta Part B, 125, 177-183, 2016.

https://doi.org/10.1016/j.sab.2016.10.001

[12] D. M. Silvestre, F. M. Barbosa, B. T. Aguiar, F. O. Leme, C. S. Nomura, "Feasibility study of calibration strategy for direct quantitative measurement of $\mathrm{K}$ and $\mathrm{Mg}$ in plant material by laser-induced breakdown spectrometry" Anal. Chem. Res.,5,28-33, 2015. https://doi.org/10.1016/j.ancr.2015.06.003

[13] E. N. Rao, P. Mathi, S. A. Kalam, S. Sreedhar, A. K. Singh, B. N. Jagatapc, S. V. Rao, "Femtosecond and nanosecond LIBS studies of nitroimidazoles: correlation between molecular structure and LIBS data" J. Anal. At. Spectrom.,31(3), 737-750, 2016 https://doi.org/10.1039/c5ja00445d

[14] S. Zivkovic, J. Savovic, M. Trtica, J. Mutic, M. Momcilovic, "Elemental analysis of aluminum alloys by Laser Induced Breakdown Spectroscopy based on TEA CO2 laser" J. Alloys Compd.,700, 175-184, 2017. https://doi.org/10.1016/j.jallcom.2017.01.060

[15] A. Matsumoto, H. Ohba, M. Toshimitsu, K. Akaoka, A. Ruas, T. Sakka, I. Wakaida, "Fiber-optic laser-induced breakdown spectroscopy of zirconium metal in air: Special features of the plasma produced by a long-pulse laser" Spectrochim. Acta Part B,142, 37-49, 2018. https://doi.org/10.1016/j.sab.2018.01.012

[16] K. Li, L. Guo, J. Li, X. Yang, R. Yi, X. Li, Y. Lu, X. Zeng, "Quantitative analysis of steel samples using laser-induced breakdown spectroscopy with an artificial neural network incorporating a genetic algorithm" Appl. Opt.,56(4), 935-941, 2017 .https://doi.org/10.1364/AO.56.000935

[17] B. N. Bennett, M. Z. Martin, D. N. Leonard, E. Garlea, Calibration curves for commercial copper and aluminum alloys using handheld laser-induced breakdown spectroscopy, App. Phys. B, 124(3):42, 2018. https://doi.org/10.1007/s00340-018-6909-x

[18] M. Momcilovic, M. Kuzmanovic, D. Rankovic, J. Ciganovic, M. Stoiljkovic, J. Savovic, M. Trticaa, "Optical Emission Studies of Copper Plasma Induced Using Infrared Transversely Excited Atmospheric (IR TEA) Carbon Dioxide Laser Pulses" Appl. Spectrosc.,69(4), 419-429. https://doi.org/10.1366/14-07584

[19] G. S. Maurya, R. Kumar, A. Kumar, A. K. Rai, "Analysis of impurities on contaminated surface of the tokamak limiter using laser induced breakdown spectroscopy" Spectrochim. Acta Part B,126, 17-22, 2016. https://doi.org/10.1016/j.sab.2016.10.010

[20] R. Ledesma, F. Palmieri, J. Connell, W. Yost, J. Fitzgerald, "Surface characterization of carbon fiber reinforced polymers by picosecond laser induced breakdown spectroscopy" Spectrochim. Acta Part B,140,5-12, (2018). https://doi.org/10.1016/j.sab.2017.11.014

[21] S. Trautner, J. Jasik, C. G. Parigger, J. D. Pedarnig, W. Spendelhofer, J. Lackner, P. Veis, J. Heitz, "Laser-induced optical breakdown spectroscopy of polymer materials based on evaluation of molecular emission bands" $\begin{array}{lllll}\text { Spectrochim. Acta Part A,174, 331-338, } 2017 . & \end{array}$ https://doi.org/10.1016/j.saa.2016.11.045

[22] P. J. Gasda, T. E. Acosta-Maeda, P. G. Lucey, A. K. Misra, S. K. Sharma, G. J. Taylor, "Next Generation Laser-Based Standoff Spectroscopy Techniques for Mars Exploration” Appl. Spectrosc.,69(2), 173-192, 2015. https://doi.org/10.1366/14-07483j.saa.2016.11.045

[23] S. Guirado, F. J. Fortes, J. J. Laserna, "Multi-Pulse Excitation for Underwater Analysis of Copper-Based Alloys Using a Novel Remote Laser-
Induced Breakdown Spectroscopy (LIBS) System" Appl. Spectrosc.,70(4), 618-626, 2016. https://doi.org/10.1177/0003702816631295

[24] X. Jiang, G. Sergienko, B. Schweer, N. Gierse, M. Hubeny, A. Kreter, S. Brezinsek, Ch. Linsmeier, Design and development of a LIBS system on linear plasma device PSI-2 for in situ real-time diagnostics of plasma-facing materials, Nucl. Mater. Energy, 12, 1224-1230, 2017. https://doi.org/10.1016/j.nme.2016.11.021

[25] S.Z. Mortazavi, P. Parvin, M. R. M. Pour, A. Reyhani, A. Moosakhani, Sh. Moradkhani, "Time-resolved evolution of metal plasma induced by Qswitched Nd:YAG and ArF-excimer lasers" Opt. Laser Technol., 62, 32-39, 2014.

https://doi.org/10.1016/j.optlastec.2014.02.006

[26] L.M. Cabalín, J.J. Laserna, "Experimental determination of laser induced breakdown thresholds of metals under nanosecond Q-switched laser operation" Spectrochim. Acta Part B, 53, 723-730, 1998. https://doi.org/10.1016/S0584-8547(98)00107-4

[27] M. A. Khater, "Laser-induced breakdown spectroscopy for light elements detection in steel: State of the art" Spectrochim. Acta Part B, 81, 1-10, 2013. https://doi.org/10.1016/j.sab.2012.12.010

[28] Y. Zhang, Y.-H. Jia, J.-W. Chen, X.-J. Shen, L. Zhao, C. Yang, Y.-Y. Chen, Y.-H. Zhang, P.-C. Han, "Study on parameters influencing analytical performance of laser-induced breakdown spectroscopy" Front. Phys. 7(6), 714-720, 2012. https://doi.org/10.1007/s11467-012-0267-7

[29] V. N.Lednev, S. M. Pershin, P. A. Sdvizhenskii, M. Y. Grishin, M. A. Davydov, A. Y. Stavertiy, R. S. Tretyakov, "Laser induced breakdown spectroscopy with picosecond pulse train" Laser Phys. Lett., 14(2), $026002,2017$. https://doi.org/10.1088/1612-202x/aa4fc0

[30] A. F. Gibson, T. P. Hughes, C. L. M. Ireland, "CO2 Laser Generation of Plasma for Spectroscopy and Spectrochemical Analysis" J. Phys. D: Appl. Phys., 4(10),1527-1534, 1971. https://doi.org/10.1088/0022-3727/4/10/310

[31] D. Colombant, G. F. Tonon, "X-Ray Emission in Laser-Produced Plasmas" J. Appl. Phys.,44(8), 3524-3537, 1973. https://doi.org/10.1063/1.1662796

[32] T. Stehrer,B. Praher,R. Viskup,J. Jasik,H. Wolfmeir,E. Arenholz,J. Heitz, J. D. Pedarnig, "Laser-induced breakdown spectroscopy of iron oxide powder" J. Anal. At. Spectrom., 24(7), 973-978, 2009. https://doi.org/10.1039/B817279J

[33] L. A. Currie, "Limits for qualitative detection and quantitative determination: Application to radiochemistry" Anal. Chem., 40(3), 586-593, 1968. https://doi.org/10.1021/ac60259a007 